\title{
Restricted Sequence Variation in Streptococcus pyogenes Penicillin Binding Proteins
}

\author{
Andrew Hayes, a Jake A. Lacey, ${ }^{\text {b Jacqueline M. Morris, }}{ }^{\text {a }}$ (D) Mark R. Davies, ${ }^{\text {a }}$ (D) Steven Y. C. Tong ${ }^{\text {b,c,d }}$ \\ aDepartment of Microbiology and Immunology, University of Melbourne, Peter Doherty Institute for Infection and Immunity, Melbourne, Victoria, Australia \\ bDoherty Department, The Peter Doherty Institute for Infection and Immunity, The University of Melbourne and The Royal Melbourne Hospital, Melbourne, Victoria, \\ Australia \\ CVictorian Infectious Disease Service, The Royal Melbourne Hospital, Peter Doherty Institute for Infection and Immunity, Melbourne, Victoria, Australia \\ dMenzies School of Health Research, Charles Darwin University, Darwin, Northern Territory, Australia
}

ABSTRACT A recent clinical report has linked Streptococcus pyogenes $\beta$-lactam antibiotic resistance to mutation in the penicillin binding protein (PBP) PBP2x. To determine whether this is an isolated case or reflects a broader prevalence of mutations that might confer reduced $\beta$-lactam susceptibility, we investigated the relative frequency of PBP sequence variation within a global database of 9,667 S. pyogenes isolates. We found that mutations in $S$. pyogenes PBPs (PBP2x, PBP1a, PBP1b, and PBP2a) occur infrequently across this global database, with fewer than 3 amino acid changes differing between $>99 \%$ of the global population. Only 4 of the 9,667 strains contained mutations near transpeptidase active sites of PBP2x or PBP1a. The reported PBP2x T553K substitution was not identified. These findings are in contrast to those of 2,520 S. pneumococcus sequences where PBP mutations are relatively frequent and are often located in key $\beta$-lactam binding pockets. These data, combined with the general lack of penicillin resistance reported in S. pyogenes worldwide, suggests that extensive, unknown constraints restrict S. pyogenes PBP sequence plasticity. Our findings imply that while heavy antibiotic pressure may select for mutations in the PBPs, there is currently no evidence of such mutations becoming fixed in the S. pyogenes population or that mutations are being sequentially acquired in the PBPs.

IMPORTANCE $\beta$-Lactam antibiotics are the first-line therapeutic option for Streptococcus pyogenes infections. Despite the global high prevalence of $S$. pyogenes infections and widespread use of $\beta$-lactams worldwide, reports of resistance to $\beta$-lactam antibiotics, such as penicillin, have been incredibly rare. Recently, $\beta$-lactam resistance, as defined by clinical breakpoints, was detected in two clinical S. pyogenes isolates with accompanying mutations in the active site of the penicillin binding protein $\mathrm{PBP} 2 \mathrm{x}$, raising concerns that $\beta$-lactam resistance will become more widespread. We screened a global database of $S$. pyogenes genome sequences to investigate the frequency of PBP mutations, identifying that PBP mutations are uncommon relative to those of Streptococcus pneumoniae. These findings support clinical observations that $\beta$-lactam resistance is rare in $S$. pyogenes and suggest that there are considerable constraints on $S$. pyogenes PBP sequence variation.

KEYWORDS Streptococcus pyogenes, beta-lactams, penicillin resistance, penicillin binding proteins

treptococcus pyogenes (group A Streptococcus, or GAS) has previously been under3 stood to be uniformly susceptible to $\beta$-lactam antibiotics (1). Two S. pyogenes isolates with elevated MICs to $\beta$-lactam antibiotics have recently been reported (2). Both isolates were molecularly typed as emm43.4 and had a penicillin binding protein
Citation Hayes A, Lacey JA, Morris JM, Davies MR, Tong SYC. 2020. Restricted sequence variation in Streptococcus pyogenes penicillin binding proteins. mSphere 5:e00090-20. https://doi.org/10.1128/mSphere.00090-20. Editor Brandi M. Limbago, U.S. Centers for Disease Control and Prevention Copyright $\odot 2020$ Hayes et al. This is an openaccess article distributed under the terms of the Creative Commons Attribution 4.0 International license.

Address correspondence to Steven Y. C. Tong, Steven.Tong@mh.org.au.

Received 28 January 2020

Accepted 20 April 2020

Published 29 April 2020 


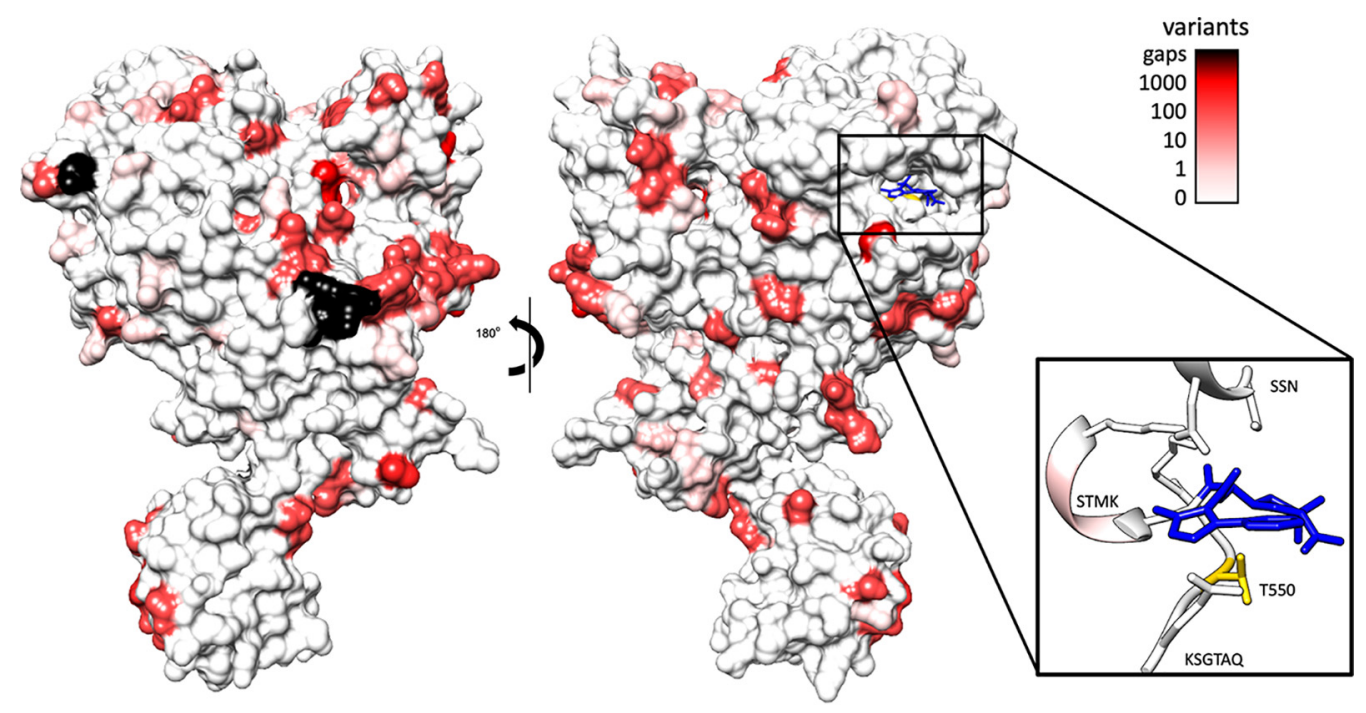

FIG 1 Global amino acid variation of Streptococcus pyogenes PBP2x mapped against the crystal structure of Streptococcus pneumoniae PBP2x. Crystal structure of PBP2x from S. pneumoniae (PDB entry 5OIZ) bound to oxacillin (blue), with the frequency of residue conservation from 9,667 S. pyogenes PBP2x sequences represented as a color gradient. Black residues represent regions absent from the alignment due to the absence of sequence relative to the $S$. pneumoniae crystal structure. Thresholds were chosen to represent differing orders of magnitude for conservation, with thresholds set at orders of magnitude $(0,1,10,100$, and 1,000 sequences varying at the residue). (Inset) Ribbon diagram of binding pocket motifs SSN, STMK, and KSG with the position of the mutated residue (T553K) highlighted (yellow). Mutations were observed in the STMK motif in 4 of the 9,667 sequences.

(PBP) PBP2x missense mutation (T553K) at the transpeptidase active site, which was associated with an 8-fold and 3-fold increased MIC to ampicillin and cefotaxime, respectively, compared to levels for closely related isolates without the PBP2x mutation. In contrast to $S$. pyogenes, reduced susceptibility to $\beta$-lactams has been widely reported in S. pneumoniae and is strongly associated with sequence variation in $\operatorname{PBPs}(3,4)$.

Using GAS genome sequences from global sources, we sought to determine the prevalence of substitutions across the transpeptidase domains of the GAS PBPs (PBP2x, PBP1a, PBP2a, and PBP1b) compared with domains of $S$. pneumoniae (which shares PBP2x and PBP1a).

\section{RESULTS}

We examined sequence variation in PBP1a, PBP1b, PBP2a, and PBP2x among 9,667 S. pyogenes genome sequences, representing 115 different emm types and 321 multilocus sequence types (see Table S1 and Text S1 to S4 in the supplemental material). These genome sequences were mostly from data sets from the United Kingdom and United States that focused on invasive disease (5-16). Mutations in the penicillin binding proteins (PBPs) have been associated with reduced clinical $\beta$-lactam susceptibility for S. pneumoniae (4), S. agalactiae (17), S. dysgalactiae (18), and now S. pyogenes (2). A comparison of PBP2x between $\beta$-lactam-susceptible reference genomes of $S$. pyogenes, S. pneumoniae, S. agalactiae, and $S$. dysgalactiae subspecies equisimilis demonstrated a high level of interspecies conservation ( $>72 \%$ similarity) (Fig. S1 and Table S2). In S. pneumoniae, substitutions at the PBP2x transpeptidase active site (SXXK, SXN, and KSTG) result in reduced $\beta$-lactam susceptibility. These three motifs were conserved across the four species (Fig. S1).

Given the similarity between PBP2x of S. pneumoniae and S. pyogenes $(73.4 \%$ similarity) (Table S1), we mapped the conservation of residues from the alignment of 9,667 S. pyogenes PBP2x sequences onto the crystal structure of S. pneumoniae PBP $2 x$ (Fig. 1). The transpeptidase active-site motifs are SXXK at positions 340 to 343 in S. pyogenes (positions 337 to 340 in S. pneumoniae), SXN at positions 399 to 401 in S. pyogenes (positions 395 to 397 in S. pneumoniae), and KSGT at positions 550 to 553 in S. pyogenes (positions 547 to 550 in S. pneumoniae). There were 101 unique amino acid 
TABLE 1 Percentage of transpeptidase sequences with variation in the SXXK, SXN, or K(T/ S)G motif of the transpeptidase active sites in PBP1a and PBP2x for S. pneumoniae and S. pyogenes

\begin{tabular}{|c|c|c|}
\hline \multirow[b]{2}{*}{ Motif } & \multicolumn{2}{|l|}{ No. (\%) of variants in: } \\
\hline & S. pneumoniae $(n=2,520)$ & S. pyogenes $(n=9,667)$ \\
\hline \multicolumn{3}{|l|}{ PBP1a } \\
\hline STMK $^{a}$ & $445(17.7)$ & $0(0)$ \\
\hline SRN & $0(0)$ & $0(0)$ \\
\hline $\mathrm{KTG}(\mathrm{T})$ & $0(0)$ & $0(0)$ \\
\hline \multicolumn{3}{|l|}{ PBP2x } \\
\hline STMK $^{a}$ & $639(25.3)$ & $4(0.04)$ \\
\hline SSN & $0(0)$ & $0(0)$ \\
\hline $\operatorname{KSG}(\mathrm{T})^{b}$ & 0 (3) [0 (0.1)] & $0(0)$ \\
\hline
\end{tabular}

aThe transpeptidase domain sequences as defined in $\mathrm{Li}$ et al. (4) were truncated between the $\mathrm{S}$ and $\mathrm{T}$ of $\mathrm{S} /$ TMK.

${ }^{b}$ The two T553K sequences reported to be associated with $\beta$-lactam resistance in S. pyogenes in Vannice et al. (2) are not included.

sequence variants of the S. pyogenes PBP $2 x$ sequence, with no frameshifts or premature stop codons (Text S1 and Table S3). We found no instances of the T553K substitution in the PBP2x KSGT motif, as reported in the recent $S$. pyogenes $\beta$-lactam-resistant isolates (2). Only four S. pyogenes isolate sequences $(0.04 \%)$ had substitutions within the transpeptidase active-site motifs of PBP2x (Fig. 1 and Table 1), corresponding to STMK to SAMK and STMK to STIK. These changes may not have a phenotypic effect on penicillin susceptibility, as STIK recently has been reported in a penicillin-susceptible isolate (GASAR0057) (16). Another 84 (0.9\%) of the 9,667 genomes contained mutations at one of four amino acid positions associated with increased tolerance to subclinical $\beta$-lactam MIC identified through a recent population genomics study of emm1, emm28, and emm89 S. pyogenes (Table S3) (19). Furthermore, no amino acid substitutions were found in the active-site motifs of S. pyogenes PBP1a. In comparison, using population data from Li et al. (4), S. pneumoniae had active-site motif variants in 639/2,520 (25.3\%) isolates for PBP2x and 445/2,520 (17.7\%) for PBP1a (Table 1). A large proportion of S. pneumoniae substitutions mapped to areas near the active site (Fig. S2).

For S. pneumoniae, the number of substitutions across the whole transpeptidase domain of PBPs has been associated with penicillin resistance. Li et al. (4) found that penicillin MICs increased as the total number of divergent (defined as $>10 \%$ amino acids different) transpeptidase domains of PBP2x, PBP1a, and PBP2b increased from 0 to 3. For S. pyogenes we used the most common amino acid sequences of PBP $2 x$ and PBP1a as our reference and, for S. pneumoniae, a previously defined wild type as the reference (4). There were considerably fewer PBP2x and PBP1a transpeptidase domains with multiple substitutions for $S$. pyogenes than for $S$. pneumoniae (Fig. 2). No $S$. pyogenes strains had sufficient mutations to reach the $10 \%$ threshold. For S. pneumoniae, $18.3 \%$ (462 of 2,520 strains) and $19.2 \%$ (485 of 2,520 strains) contained divergent PBP2x and PBP1a transpeptidase domains, respectively (Fig. 2). This pattern of greater conservation of S. pyogenes PBPs was also observed for PBP1b and PBP2a in S. pyogenes compared to PBP2b in S. pneumoniae (Fig. S3).

\section{DISCUSSION}

Expanding on recent findings (19), we found no evidence that mutations are present in the $\beta$-lactam binding site KSGTAQ motif of PBP2x among 9,667 genetically and geographically diverse $S$. pyogenes genome sequences. While sporadic mutations were observed in PBP proteins, only four isolates contained mutations in the transpeptidase active sites of PBP2x and PBP1a. A further 84 strains $(<1 \%)$ carried PBP2x amino acid variations recently associated with an increased tolerance to subclinical penicillin MIC (19). Although the report of two $S$. pyogenes isolates with clinical $\beta$-lactam resistance associated with $p b p 2 x$ mutations is concerning (2), our findings provide reassurance that PBP mutations leading to clinical resistance are extremely limited, and perhaps 

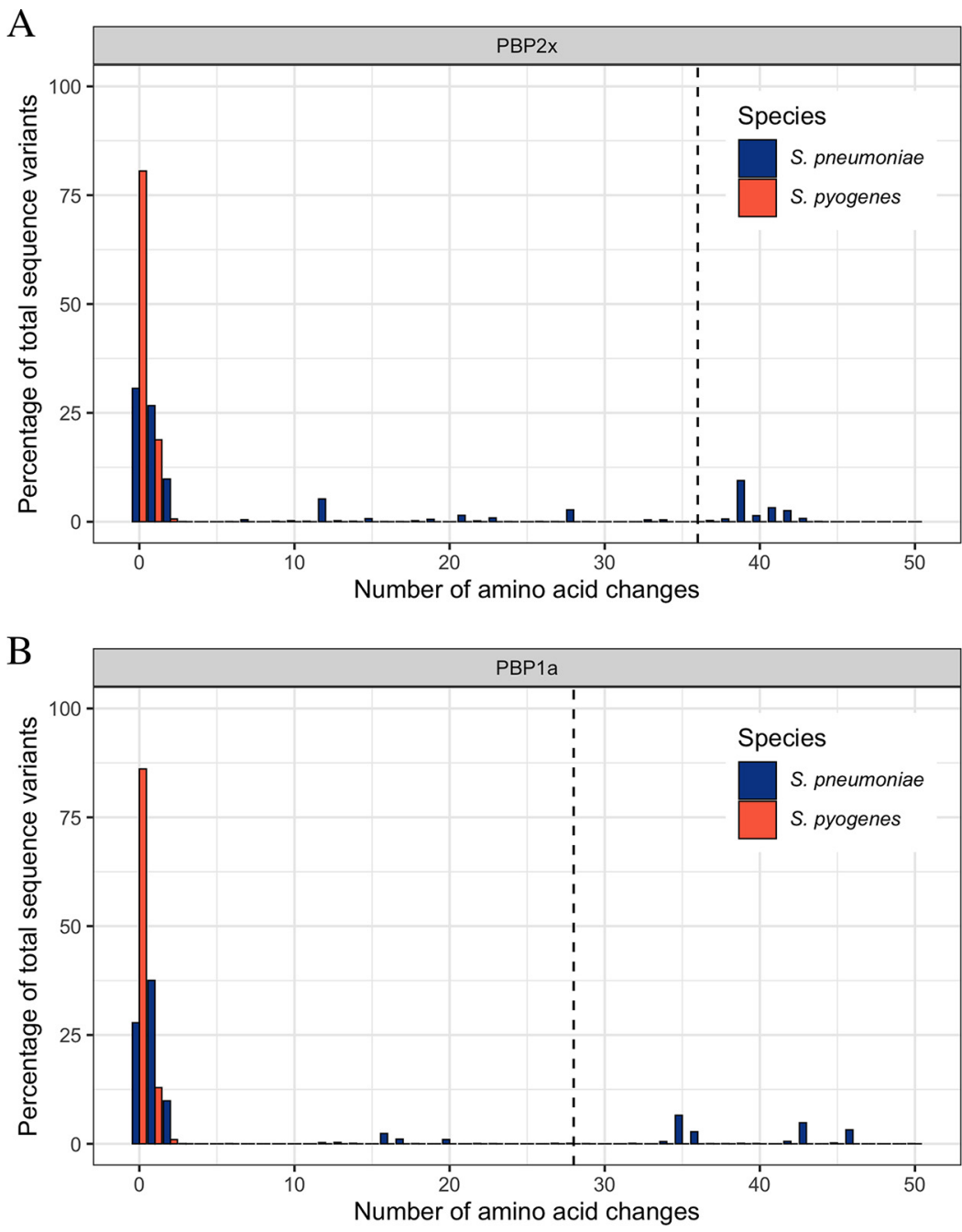

FIG 2 Amino acid differences of the transpeptidase domains of PBP2x and PBP1a. (A and B) The percentage of isolates with changes in the transpeptidase domains of PBP2X (A) and PBP1a (B) relative to penicillin-susceptible references in Streptococcus pneumoniae (blue; $n=2,520$ ) and S. pyogenes (red; $n=9,667$ ). Sequences that are $>10 \%$ divergent (indicated by dotted vertical lines) have been associated with increased penicillin MICs in S. pneumoniae.

unique, occurrences at this stage. Similar observations have been reported within closely related beta-hemolytic streptococci such as Streptococcus agalactiae and Streptococcus dysgalactiae subspecies equisimilis, where PBP mutations conferring reduced penicillin susceptibility or resistance were observed $(18,20)$, but without conclusive evidence of clonal expansion through current population-based surveillance investigations $(6,20)$.

We found a high degree of conservation of GAS PBP2x and PBP1a at transpeptidase active sites and across the broader transpeptidase domains. In comparison, PBP2x and PBP1a for S. pneumoniae were far less conserved, suggesting that there are strong evolutionary constraints in these domains for $S$. pyogenes that is not the case for $S$. pneumoniae. This may be due to several factors, including the lack of structural plasticity possible in PBP proteins of GAS (S. pyogenes lacks a PBP2b homolog), different $\beta$-lactam-resistant communities within the environmental niches occupied, lower natural transformation efficiency of GAS relative to that of S. pneumoniae, and a necessity for other chromosomal compensatory mutations to facilitate the maintenance of clinically relevant PBP mutations, as has been suggested for group B streptococci (2). Studies of penicillin-resistant $S$. pyogenes generated through mutagenesis (21) or serial 
passage in penicillin-containing medium (22) demonstrated that mutants with raised penicillin MICs appeared to have alterations in PBPs with reduced penicillin affinity (21). Notably, mutants grow more slowly, have aberrant colony morphology compared to that of wild-type strains (21), and are avirulent, with a decrease in M protein production (22). These laboratory experiments, together with the absence of naturally occurring isolates with greater than five amino acid substitutions in PBP2x or PBP1a, strongly suggest that changes to the PBPs are associated with a significant fitness cost. However, as subclinical low-level $\beta$-lactam resistance theoretically could confer biological advantages to $S$. pyogenes carriage, maintaining vigilance through population-based $S$. pyogenes surveillance for PBP variants is encouraged (19).

\section{MATERIALS AND METHODS}

We obtained publicly available genome sequence data for 9,667 S. pyogenes isolates from the short-read archive (see Table S1 in the supplemental material). We assembled genomes using shovill v.1.0.9 (https://github.com/tseemann/shovill) with an underlying SKESA v.2.3.0 assembler (23). Using the $\beta$-lactam-susceptible $S$. pyogenes serotype M3 strain ATCC BAA-595/MGAS315 as a reference, we determined the presence, amino acid sequence, and alignment (24) of each of PBP2x, PBP1a, PBP1b, and PBP2a in each genome with the screen_assembly script (5) and BLASTP parameters of 100\% coverage and $90 \%$ identity. Variant sites were identified from the multi-FASTA alignments using snp-sites (25).

To compare the conservation of the transpeptidase active-site motifs across streptococcal species, full-length PBP2x protein sequences of $S$. pyogenes serotype M3 strain ATCC BAA-595/MGAS315 (GenBank accession no. NC_004070.1), S. pneumoniae strain ATCC BAA-255/R6 (NC_003098.1), S. agalactiae strain 2603V/R (NC_004116.1), and the S. dysgalactiae subspecies equisimilis strain RE378 (NC_018712.1) reference genomes were aligned using Clustal Omega $(26,27)$. The percent sequence similarity was compared using Blosum62 with a threshold of 1 in Geneious Prime (28).

To investigate the inferred crystal structure location of $S$. pyogenes PBP2x mutations relative to that of the S. pneumoniae orthologue, S. pyogenes PBP2x sequence variations were plotted onto the $S$. pneumoniae PBP2x crystal structure bound to oxacillin (PDB entry 5OIZ) (29). Sequence conservation, as determined by the frequency (for S. pyogenes) and percentage (for S. pneumoniae) of variant amino acids compared to the consensus, was rendered onto the PBP2x crystal structure using UCSF Chimera (30).

We defined the PBP2x and PBP1a transpeptidase regions as those used in an assessment of 2,520 invasive S. pneumoniae isolates by Li et al. (4) and determined and plotted the number of pairwise amino acid differences within these regions using Distances Matrix in Geneious Prime (28) and ggplot2 in R version 3.6.1 (31). Similarly, we also assessed the conservation of PBP1b and PBP2a proteins for the 9,667 S. pyogenes genomes and the transpeptidase region of PBP2b for S. pneumoniae.

\section{SUPPLEMENTAL MATERIAL}

Supplemental material is available online only.

TEXT S1, TXT file, $0.2 \mathrm{MB}$.

TEXT S2, TXT file, $0.2 \mathrm{MB}$.

TEXT S3, TXT file, $0.2 \mathrm{MB}$.

TEXT S4, TXT file, $0.2 \mathrm{MB}$.

FIG S1, PDF file, $0.05 \mathrm{MB}$.

FIG S2, PDF file, $1 \mathrm{MB}$.

FIG S3, PDF file, $0.2 \mathrm{MB}$.

TABLE S1, XLSX file, $0.4 \mathrm{MB}$.

TABLE S2, DOCX file, $0.02 \mathrm{MB}$.

TABLE S3, DOCX file, $0.03 \mathrm{MB}$.

\section{ACKNOWLEDGMENTS}

This work was supported by Australian National Health and Medical Research Council (NHMRC) project grant number 1098319. S.Y.C.T. is an NHMRC Career Development Fellow (number 1145033). M.R.D. is the recipient of a University of Melbourne C. R. Roper Fellowship.

The funders had no role in study design, data collection and interpretation, or the decision to submit the work for publication.

\section{REFERENCES}

1. Horn DL, Zabriskie JB, Austrian R, Cleary PP, Ferretti JJ, Fischetti VA, Gotschlich E, Kaplan EL, McCarty M, Opal SM, Roberts RB, Tomasz A, Wachtfogel Y. 1998. Why have group A streptococci remained suscep- tible to penicillin? Report on a symposium. Clin Infect Dis 26:1341-1345. https://doi.org/10.1086/516375

2. Vannice K, Ricaldi J, Nanduri S, Fang FC, Lynch J, Bryson-Cahn C, Wright 
T, Duchin J, Kay M, Chochua S, Van Beneden C, Beall B. 2019. Streptococcus pyogenes $p b p 2 x$ mutation confers reduced susceptibility to betalactam antibiotics. Clin Infect Dis 70:1265. https://doi.org/10.1093/cid/ ciz1000.

3. Dewe TCM, D'Aeth JC, Croucher NJ. 2019. Genomic epidemiology of penicillin-non-susceptible Streptococcus pneumoniae. Microb Genom 5:e000305. https://doi.org/10.1099/mgen.0.000305.

4. Li Y, Metcalf BJ, Chochua S, Li Z, Gertz RE, Jr, Walker H, Hawkins PA, Tran T, Whitney CG, McGee L, Beall BW. 2016. Penicillin-binding protein transpeptidase signatures for tracking and predicting beta-lactam resistance levels in Streptococcus pneumoniae. mBio 7:e00756-16. https://doi .org/10.1128/mBio.00756-16.

5. Davies MR, McIntyre L, Mutreja A, Lacey JA, Lees JA, Towers RJ, Duchene S, Smeesters PR, Frost HR, Price DJ, Holden MTG, David S, Giffard PM Worthing KA, Seale AC, Berkley JA, Harris SR, Rivera-Hernandez T, Berking $O$, Cork AJ, Torres R, Lithgow T, Strugnell RA, Bergmann R, NitscheSchmitz P, Chhatwal GS, Bentley SD, Fraser JD, Moreland NJ, Carapetis JR, Steer AC, Parkhill J, Saul A, Williamson DA, Currie BJ, Tong SYC, Dougan G, Walker MJ. 2019. Atlas of group A streptococcal vaccine candidates compiled using large-scale comparative genomics. Nat Genet 51:1035-1043. https://doi.org/10.1038/s41588-019-0417-8.

6. Athey TB, Teatero S, Sieswerda LE, Gubbay JB, Marchand-Austin A, Li A Wasserscheid J, Dewar K, McGeer A, Williams D, Fittipaldi N. 2016. High incidence of invasive group A Streptococcus disease caused by strains of uncommon emm types in Thunder Bay, Ontario, Canada. J Clin Microbiol 54:83-92. https://doi.org/10.1128/JCM.02201-15

7. Ben Zakour NL, Davies MR, You Y, Chen JH, Forde BM, Stanton-Cook M, Yang R, Cui Y, Barnett TC, Venturini C, Ong CL, Tse H, Dougan G, Zhang J, Yuen KY, Beatson SA, Walker MJ. 2015. Transfer of scarlet feverassociated elements into the group A Streptococcus M1T1 clone. Sci Rep 5:15877. https://doi.org/10.1038/srep15877.

8. Bergin SM, Periaswamy B, Barkham T, Chua HC, Mok YM, Fung DSS, Su AHC, Lee YL, Chua MLI, Ng PY, Soon WJW, Chu CW, Tan SL, Meehan M, Ang BSP, Leo YS, Holden MTG, De P, Hsu LY, Chen SL, de Sessions PF, Marimuthu K. 2018. An outbreak of Streptococcus pyogenes in a mental health facility: advantage of well-timed whole-genome sequencing over emm typing. Infect Control Hosp Epidemiol 39:852-860. https://doi.org/ 10.1017/ice.2018.101.

9. Chalker V, Jironkin A, Coelho J, Al-Shahib A, Platt S, Kapatai G, Daniel R, Dhami C, Laranjeira M, Chambers T, Guy R, Lamagni T, Harrison T, Chand M, Johnson AP, Underwood A, Scarlet Fever Incident Management Team. 2017. Genome analysis following a national increase in scarlet fever in England 2014. BMC Genomics 18:224. https://doi.org/10.1186/ s12864-017-3603-z.

10. Chochua S, Metcalf BJ, Li Z, Rivers J, Mathis S, Jackson D, Gertz RE, Jr, Srinivasan V, Lynfield R, Van Beneden C, McGee L, Beall B. 2017. Population and whole genome sequence based characterization of invasive group A streptococci recovered in the United States during 2015. mBio 8:e01422-17. https://doi.org/10.1128/mBio.01422-17.

11. Coelho JM, Kapatai G, Jironkin A, Al-Shahib A, Daniel R, Dhami C, Laranjeira AM, Chambers T, Phillips S, Tewolde R, Underwood A, Chalker VJ. 2019. Genomic sequence investigation Streptococcus pyogenes clusters in England (2010-2015). Clin Microbiol Infect 25:96-101. https://doi .org/10.1016/j.cmi.2018.04.011.

12. Davies MR, Holden MT, Coupland P, Chen JH, Venturini C, Barnett TC, Zakour NL, Tse H, Dougan G, Yuen KY, Walker MJ. 2015. Emergence of scarlet fever Streptococcus pyogenes emm12 clones in Hong Kong is associated with toxin acquisition and multidrug resistance. Nat Genet 47:84-87. https://doi.org/10.1038/ng.3147.

13. Dickinson $H$, Reacher $M$, Nazareth $B$, Eagle $H$, Fowler D, Underwood $A$, Chand M, Chalker V, Coelho J, Daniel R, Kapatai G, Al-Shabib A, Puleston R. 2019. Whole-genome sequencing in the investigation of recurrent invasive group A Streptococcus outbreaks in a maternity unit. J Hosp Infect 101:320-326. https://doi.org/10.1016/j.jhin.2018.03.018.

14. Kapatai G, Coelho J, Platt S, Chalker VJ. 2017. Whole genome sequencing of group A Streptococcus: development and evaluation of an automated pipeline for emm gene typing. PeerJ 5:e3226. https://doi.org/10.7717/ peerj.3226.

15. Lynskey NN, Jauneikaite E, Li HK, Zhi X, Turner CE, Mosavie M, Pearson M, Asai M, Lobkowicz L, Chow JY, Parkhill J, Lamagni T, Chalker VJ, Sriskandan S. 2019. Emergence of dominant toxigenic M1T1 Streptococcus pyogenes clone during increased scarlet fever activity in England: a population-based molecular epidemiological study. Lancet Infect Dis 19:1209-1218. https://doi.org/10.1016/S1473-3099(19)30446-3.

16. Turner CE, Bedford L, Brown NM, Judge K, Torok ME, Parkhill J, Peacock SJ. 2017. Community outbreaks of group A Streptococcus revealed by genome sequencing. Sci Rep 7:8554. https://doi.org/10.1038/s41598-017 -08914-x.

17. Dahesh S, Hensler ME, Van Sorge NM, Gertz RE, Jr, Schrag S, Nizet V, Beall BW. 2008. Point mutation in the group B streptococcal pbp2x gene conferring decreased susceptibility to beta-lactam antibiotics. Antimicrob Agents Chemother 52:2915-2918. https://doi.org/10.1128/AAC .00461-08

18. Fuursted K, Stegger M, Hoffmann S, Lambertsen L, Andersen PS, Deleuran M, Thomsen MK. 2016. Description and characterization of a penicillin-resistant Streptococcus dysgalactiae subsp. equisimilis clone isolated from blood in three epidemiologically linked patients. J Antimicrob Chemother 71:3376-3380. https://doi.org/10.1093/jac/dkw320.

19. Musser JM, Beres SB, Zhu L, Olsen RJ, Vuopio J, Hyyrylainen $\mathrm{HL}_{\text {, }}$ Grondahl-Yli-Hannuksela K, Kristinsson KG, Darenberg J, HenriquesNormark B, Hoffmann S, Caugant DA, Smith AJ, Lindsay DSJ, Boragine DM, Palzkill T. 2020. Reduced in vitro susceptibility of Streptococcus pyogenes to beta-lactam antibiotics associated with mutations in the pbp2x gene is geographically widespread. J Clin Microbiol 58:e01993-19. https://doi.org/10.1128/JCM.01993-19.

20. Metcalf BJ, Chochua S, Gertz RE, Jr, Hawkins PA, Ricaldi J, Li Z, Walker H, Tran T, Rivers J, Mathis S, Jackson D, Glennen A, Lynfield R, McGee L, Beall B, Active Bacterial Core Surveillance Team. 2017. Short-read whole genome sequencing for determination of antimicrobial resistance mechanisms and capsular serotypes of current invasive Streptococcus agalactiae recovered in the USA. Clin Microbiol Infect 23:574.e7-574.e14. https://doi.org/10.1016/j.cmi.2017.02.021.

21. Gutmann L, Tomasz A. 1982. Penicillin-resistant and penicillin-tolerant mutants of group A streptococci. Antimicrob Agents Chemother 22: 128-136. https://doi.org/10.1128/aac.22.1.128.

22. Rosendal K. 1958. Investigations of penicillin-resistant streptococci belonging to group A. Acta Pathol Microbiol Scand 42:181-188. https:// doi.org/10.1111/j.1699-0463.1958.tb03183.x.

23. Souvorov A, Agarwala R, Lipman DJ. 2018. SKESA: strategic k-mer extension for scrupulous assemblies. Genome Biol 19:153. https://doi.org/ 10.1186/s13059-018-1540-z.

24. Edgar RC. 2004. MUSCLE: a multiple sequence alignment method with reduced time and space complexity. BMC Bioinformatics 5:113. https:// doi.org/10.1186/1471-2105-5-113.

25. Page AJ, Taylor B, Delaney AJ, Soares J, Seemann T, Keane JA, Harris SR. 2016. SNP-sites: rapid efficient extraction of SNPs from multi-FASTA alignments. Microb Genom 2:e000056. https://doi.org/10.1099/mgen.0 .000056 .

26. Goujon M, McWilliam H, Li W, Valentin F, Squizzato S, Paern J, Lopez R. 2010. A new bioinformatics analysis tools framework at EMBL-EBI. Nucleic Acids Res 38:W695-W699. https://doi.org/10.1093/nar/gkq313.

27. Sievers F, Wilm A, Dineen D, Gibson TJ, Karplus K, Li W, Lopez R, McWilliam H, Remmert M, Soding J, Thompson JD, Higgins DG. 2011. Fast, scalable generation of high-quality protein multiple sequence alignments using Clustal Omega. Mol Syst Biol 7:539. https://doi.org/10.1038/msb.2011.75.

28. Kearse M, Moir R, Wilson A, Stones-Havas S, Cheung M, Sturrock S, Buxton S, Cooper A, Markowitz S, Duran C, Thierer T, Ashton B, Meintjes P, Drummond A. 2012. Geneious Basic: an integrated and extendable desktop software platform for the organization and analysis of sequence data. Bioinformatics 28:1647-1649. https://doi.org/ 10.1093/bioinformatics/bts199.

29. Bernardo-Garcia N, Mahasenan KV, Batuecas MT, Lee M, Hesek D, Petrackova D, Doubravova L, Branny P, Mobashery S, Hermoso JA. 2018. Allostery, recognition of nascent peptidoglycan, and cross-linking of the cell wall by the essential penicillin-binding protein $2 x$ of Streptococcus pneumoniae. ACS Chem Biol 13:694-702. https://doi.org/10.1021/acschembio .7b00817.

30. Pettersen EF, Goddard TD, Huang CC, Couch GS, Greenblatt DM, Meng EC, Ferrin TE. 2004. UCSF Chimera-a visualization system for exploratory research and analysis. J Comput Chem 25:1605-1612. https://doi.org/10 .1002/jcc.20084.

31. R Core Team. 2019. R: a language and environment for statistical computing. R Foundation for Statistical Computing, Vienna, Austria. https:// www.R-project.org. 


\section{University Library}

\section{- M M I N E R VA A gateway to Melbourne's research publications}

Minerva Access is the Institutional Repository of The University of Melbourne

\section{Author/s:}

Hayes, A;Lacey, JA;Morris, JM;Davies, MR;Tong, SYC

Title:

Restricted Sequence Variation in Streptococcus pyogenes Penicillin Binding Proteins

\section{Date:}

2020-03-01

Citation:

Hayes, A., Lacey, J. A., Morris, J. M., Davies, M. R. \& Tong, S. Y. C. (2020). Restricted

Sequence Variation in Streptococcus pyogenes Penicillin Binding Proteins. MSPHERE, 5 (2), https://doi.org/10.1128/mSphere.00090-20.

Persistent Link:

http://hdl.handle.net/11343/246306

License:

CC BY 